\title{
Ventral Nasal Turbinate
}

National Cancer Institute

\section{Source}

National Cancer Institute. Ventral Nasal Turbinate. NCI Thesaurus. Code C139165.

The nasal turbinate originating from the conchal crest on the medial wall of the maxilla and extending into the nasal cavity. 